\title{
Transient Global Amnesia Associated with Acute Intracerebral Hemorrhage at the Cingulate Gyrus
}

\author{
Bora Yoon Ji-Yeon Yoo Yong-Soo Shim Kwang-Soo Lee Joong-Seok Kim \\ Department of Neurology, The Catholic University of Korea, Seoul, Korea
}

Dear Sir,

Transient global amnesia (TGA) is a benign neurological syndrome characterized by temporary loss of anterograde and recent retrograde memory with preserved consciousness and self-awareness; it is usually accompanied by repetitive questioning and temporal disorientation for a limited period of time [1]. In the majority of TGA cases, no cause can be identified; structural imaging is invariably normal and CSF studies are generally not performed. EEGs that have been performed during an episode have been reported to demonstrate no epileptiform abnormalities [2]. Several different causes for this syndrome have been proposed, including ischemia, migraine, epileptic seizure, venous congestion and psychological disturbances; however, there remains no consensus on the etiology [3]. Moreover, TGA associated with intracerebral hemorrhage has rarely been reported.

The patient described here presented with transient amnesia resulting from a left cingulate lesion. This is of particular interest because these findings suggest that activity in the cingulate gyrus and its outflow pathways may lead to TGA.

\section{Case Report}

A 57-year-old right-handed man was admitted via the emergency room because of an acute memory disturbance and repetitive questioning. He asked his friend the same questions repeatedly, e.g. 'What am I doing here?', 'How did I get here?'. When his friend gave him an answer, he then said 'Yes, I see', but then repeated a similar question again. He did not complain of any other neurological symptoms such as headache, dizziness or focal weakness. There was no history of hypertension, diabetes, transient ischemic attacks, stroke, epilepsy, migraine, psychiatric illness, head trauma or alcohol abuse.

On examination, the patient was alert, with a blood pressure of 130/80 $\mathrm{mm} \mathrm{Hg}$, a regular heart beat of 75 beats/min and a temperature of $36.7^{\circ} \mathrm{C}$. Physical examination demonstrated no pathologic findings. On cognitive function evaluation, comprehension was intact and the patient was fully conscious; however, he immediately forgot what was heard and continued repetitive questioning regarding what he was doing here. Except for memory deficits, the other cognitive profiles, which included attention, praxis, language and frontal function, were normal. The patient's amnesia and repetitive questioning gradually resolved after $6 \mathrm{~h}$. The next morning, the patient was oriented and had recovered anterograde and recent retrograde memory, but not memory during the attack.

Laboratory studies including complete blood cell and platelet count, erythrocyte sedimentation rate, blood electrolytes, creatinine, liver enzymes, cholesterol, triglycerides, prothrombin and partial thromboplastin time, antithrombin III-protein C and protein $S$ activity were all normal. In addition, autoantibody screens were also normal. Neither the electrocardiogram from a 24-hour Holter monitor nor transthoracic echocardiography revealed any cardiac abnormalities. The waking surface EEG during the episode was normal. Brain
MRI studies 3 days after the attack, including gradient echo images, demonstrated a focal intracerebral hemorrhage at the left cingulate gyrus. The hippocampus, thalamus and basal ganglia appeared normal. Magnetic resonance angiograms of the brain were also normal (fig. 1).

A neuropsychological test for memory function performed 7 days after the episode was within normal limits. The Korean Mini-Mental State Examination score was 29. On the 12-word list test from the Hopkins Verbal Learning Test for verbal memory the scores for immediate recall were 5 in the first trial, 7 in the second trial and 8 in the third trial; the result after a 20 -min delayed recall was 6 , and the recognition score was 9 (true positive 10 and false positive 1). The results of the Digit Span Test for attention and immediate memory were Forward 7 and Backward 5. The score for the Korean version of the Boston Naming Test for language was 51, and the scores for the Word Fluency Test (animal, grocery item and 3 Korean letters) were 15, 17, 6, 7, 7 , respectively. The Go/No Go tasks and the Luria Loop Test for frontal lobe function were normal. Positron emission topography scans using ${ }^{18} \mathrm{~F}$-dexoyglucose performed on the same day did not reveal any abnormalities.

\section{Discussion}

Explanation of the mechanisms involved in a TGA have included the hypoxic-ischemic theory, which might best explain the findings of high prevalence of emotional distress, personality traits, and

\section{KARGER}

Fax +41613061234 E-Mail karger@karger.ch www.karger.com
(C) 2006 S. Karger AG, Basel 0014-3022/06/0561-0054\$23.50/0

Accessible online at: www.karger.com/ene
Joong-Seok Kim, MD

Department of Neurology, Kangnam St. Mary Hospital

505, Banpo-dong, Seocho-gu

Seoul, 137-070 (Korea)

Tel. +82 2590 2091, Fax +82 2599 9686, E-Mail neuronet@catholic.ac.kr 

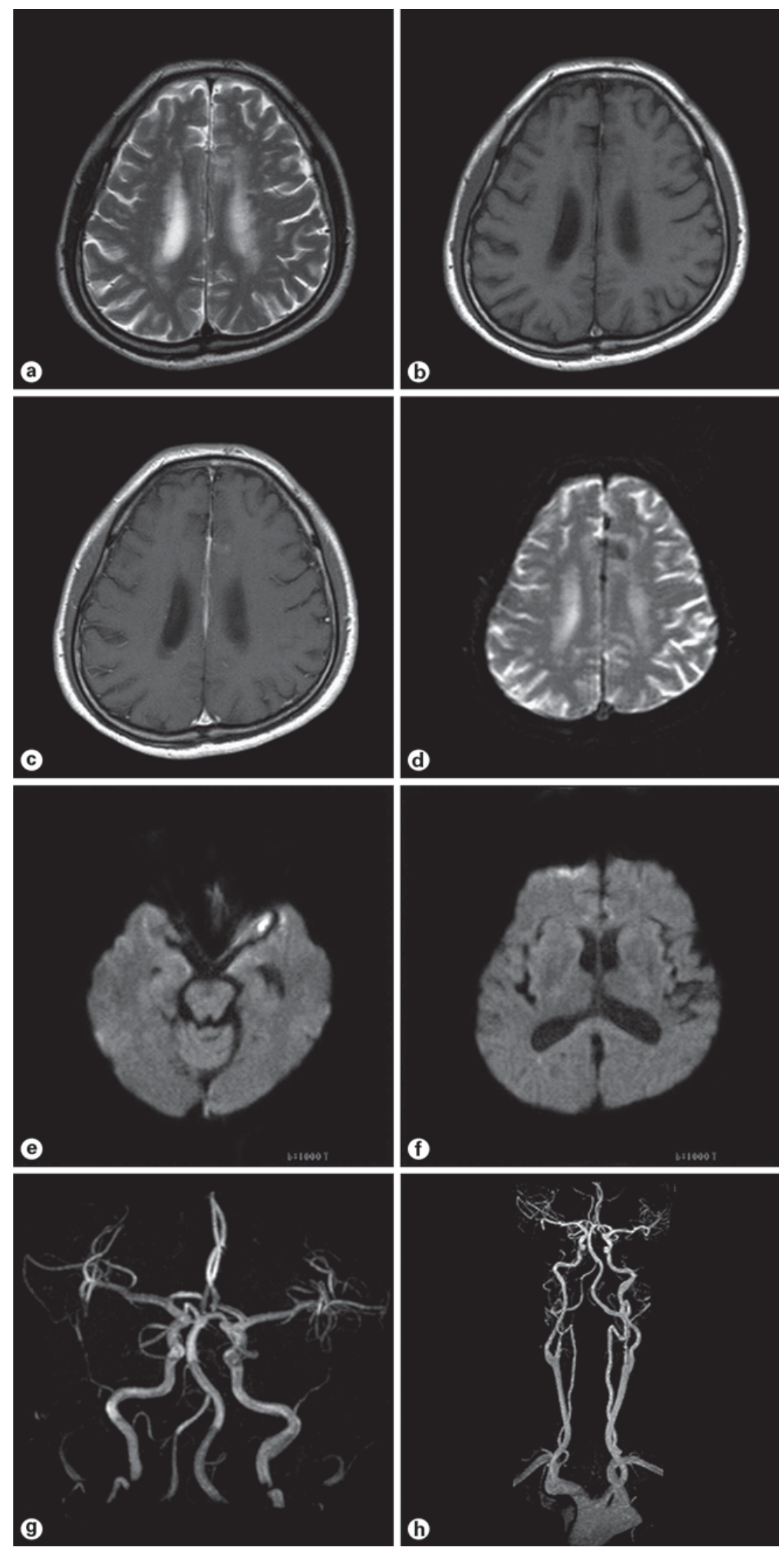

Fig. 1. Hemorrhage was found at the left cingulated gyrus on the $\mathrm{T}_{2}$-weighted axial image (a), $\mathrm{T}_{1}$-weighted axial image (b), $\mathrm{T}_{1}$-enhanced axial image (c), and gradient echo image (d). e, f The hippocampus, thalamus and basal ganglia appeared normal on diffusion-weighted images. $\mathbf{g}$, h Magnetic resonance angiograms of the brain were also normal. 
Valsalva-like activities before onset of symptoms as well as a high frequency of cerebral infarction and ischemic changes found on brain imaging $[4,5]$. However, there are some reports that argue against an ischemic etiology for TGA that have shown a lack of association between vascular risk factors and TGA, and signal changes on DWI from nonischemic causes [6]. Patients with intracerebral hemorrhage presenting with TGA have also been reported, but rarely. Some investigators have proposed a cortical spreading depression as the cause of TGA. In a report by Strupp et al. [7], description of widespread transient hyperintensities in the left or bilateral mesial temporal lobes was observed in patients with acute TGA. The researchers believed that these findings were consistent with a spreading depression. Additionally, Strong et al. [8] reported cortical spreading and synchronous depression on electrocorticography in patients after head injury or intracranial hemorrhage.

Despite the unresolved etiology of TGA, there is general agreement that pathological changes affect the mediobasal temporal region, the hippocampus and the parahippocampus. It has been reported that TGA occurred in patients with acute infarction of the hippocampus, thalamus, caudate nucleus [9] and retrosplenium of the corpus callosum [10], or with hemorrhage at the thalamus, lenticular nucleus [11], and frontal lobe [12]. Studies utilizing functional neuroimaging observations suggest a dysfunction in memory-relevant structures such as the prefrontal cortex, hippocampus, temporal lobe or thalamus during the acute stage of TGA [13-19]. These findings suggest that all loci identified have been within the areas of memory-relevant structures including the Papez circuit, and most have been on the left side of the brain.

Our patient presented with a TGA which was related to a small hemorrhage neighboring the left cingulate gyrus. The findings from this case support that the cingulate cortex, including the cingulum which connects to the entorhinal, prefrontal cortex and other neocortical areas, may be important to the memory circuit (Papez circuit) and critical to the maintenance and processing of cognitive function [20, 21].
It is of course possible that TGA may be a coincidental finding in a patient with a cingulate hemorrhage. However, the relationship between clinical TGA and the subacute-stage hemorrhage of cingulate gyrus, as well as the absence of other observed etiologies, favors a cause-and-effect relationship, rather than a coincidental occurrence.

In our patient, the involvement of the cingulate gyrus was thought to be due to a wide range of possible causes, because the etiology remains unclear and the lesion location was uncommon. The differential diagnosis may include superior sagittal sinus thrombosis, subarachnoid hemorrhage, vasculitides and hypertensive encephalopathy. However, these diseases can be excluded in our case due to the clinical presentation, normal laboratory findings and absence of typical radiological signs such as an empty delta.

In conclusion, although there remains no consensus on the etiology of TGA, any focal involvement of a memory-related structure may cause TGA. This case may aid in our understanding of the relationship between a lesion and the etiology of TGA, despite the fact that a cause-and-effect relationship cannot be proven.

\section{References}

1 Fisher CM, Adams RD: Transient global amnesia. Acta Neurol Scand 1964;40:S1-S83.

2 Jacome DE: EEG features in transient global amnesia. Clin Electroenceph 1989;20:183192.

3 Pantoni L, Lamassa M, Inzitari D: Transient global amnesia: a review emphasizing pathogenic aspects. Acta Neurol Scand 2000;102: 275-83.

4 Ay H, Fruie KL, Yamada K, Korroshetz WJ: Diffusion-weighted MRI characterize the ischemic lesion in transient global amnesia. Neurology 1998;51:901-903.

5 Santos S, Lopez del Val J, Tejero C, Inguez C, Lalana JM, Morales F: Transient global amnesia: a review of 58 cases. Rev Neurol 2000; 30:1113-1117.

6 Gass A, Gaa J, Hirsch J, Schwartz A, Hennerici MG: Lack of evidence of ischemic tissue change in transient global amnesia on single-shot echo-planar diffusion-weighted MRI. Stroke 1999;30:2070-2072.

7 Strupp M, Bruning R, Wu RH, Deimling M, Reiser M, Brandt T: Diffusion-weighted MRI in transient global amnesia: elevated signal intensity in the left mesial temporal lobe in 7 of 10 patients. Ann Neurol 1998;43:164-170.
8 Strong AJ, Fabricius M, Boutelle MG, et al: Spreading and synchronous depression of cortical activity in acutely injured human brain. Stroke 2002;33:2738-2743.

9 Ravindran V, Jain S, Ming A, Bartlett RJ: Transient global amnesia in a patient with acute unilateral caudate nucleus ischemia. J Clin Neurosci 2004;11:669-672.

10 Saito K, Kimura K, Minematsu K, Shiraishi A, Nakajima M: Transient global amnesia associated with an acute infarction in the retrosplenium of the corpus callosum. J Neurol Sci 2003;210:95-97.

11 Bogousslavsky J, Regli F: Transient global amnesia and stroke. Eur Neurol 1988;28: 106-110.

12 Jacome DE, Yanez GF: Transient global amnesia and left frontal hemorrhage. Postgrad Med J 1988;64:137-139.

13 Warren JD, Chatterton B, Thompson PD: A SPECT study of the anatomy of transient global amnesia. J Clin Neurosci 2000;10:5759.

14 Schmidtke K, Reinhardt M, Krause T: Cereral perfusion during transient global amnesia: findings with HMPAO SPECT. J Nucl Med 1998;39:155-159.

15 Laloux P, Brichant C, Cauwe F, Decoster P: Technetium-99m HMPAO single photon emission computed tomography imaging in transient global amnesia. Arch Neurol 1992; 49:543-546.

16 Fujii K, Sadoshima S, Ishitsuka T, Kusuda K, Kuwabara Y, Ichiya Y, Fujishima M: Regional cerebral blood flow and metabolism in patients with transient global amnesia: a positron emission tomography study. J Neurol Neurosurg Psychiatry 1989;52:622-630.

17 Eustache F, Desgranges B, Aupee AM, Guillery B, Baron JC: Functional neuroanatomy of amnesia: positron emission tomography studies. Microsc Res Tech 2000;51:94-100.

18 Baron JC, Petit-Taboue MC, Le Doze F, Desgranges B, Ravenel N, Marchal G: Right frontal cortex hypometabolism in transient global amnesia. A PET study. Brain 1994;117: 545-552.

19 Fazio F, Perani D, Gilardi MC, Colombo F, Cappa SF, Vallar G, et al: Metabolic impairment in human amnesia: a PET study of memory networks. J Cereb Blood Flow Metab $1992 ; 12: 353-358$.

20 Yoshiura T, Mihara F, Ogomori K, Tanaka A, Kaneko K, Masuda K: Diffusion tensor in posterior cingulate gyrus: correlation with cognitive decline in Alzheimer's disease. Neuroreport 2002;13:2299-2302.

21 Cammalleri R, Gangitano M, D’Amelio M, Raieli V, Raimondo D, Camarda R: Transient topographical amnesia and cingulate cortex damage: a case report. Neuropsychologia 1996;34:321-326. 